\title{
Robotic excision of pulmonary sclerosing pneumocytoma: a case report
}

\author{
Matthew O. Smith ${ }^{1}$, Sabrina L. Mason ${ }^{2}$, Dileep Duvva ${ }^{1}$, Steven M. Woolley ${ }^{1}$ \\ ${ }^{1}$ Department of Cardiothoracic Surgery, Liverpool Heart and Chest Hospital, Liverpool, UK; ${ }^{2}$ School of Medicine, University of Liverpool, \\ Liverpool, UK \\ Correspondence to: Matthew O. Smith. Department of Cardiothoracic Surgery, Liverpool Heart and Chest Hospital, Thomas Drive, Liverpool, L14 \\ 3PE, UK. Email: Matthew.Smith@1hch.nhs.uk.
}

\begin{abstract}
We report the first excision of pulmonary sclerosing pneumocytoma (PSP) via a robotic approach. A 20-year-old Caucasian male presented with an incidental $48 \mathrm{~mm}$ mass in the right lower lobe. Computerized tomography (CT)-guided biopsy was performed achieving a pre-operative diagnosis of PSP, previously known as pulmonary sclerosing hemangioma. A key histological feature of PSP is the presence of both cuboidal surface cells and stromal round cells and this results in a challenge in diagnosing them on frozen section where they can be easily mistaken for adenocarcinoma or carcinoid tumours. With most PSPs typically presenting as a solitary peripheral nodule, the differential diagnosis is wide. On CT, a predominantly smooth boundary and oval shape is common. The varying proportions of the haemangiomatous, papillary, sclerotic and solid histological patterns result in varying density of 96 to 157 Hounsfield Units, with higher attenuation in angiomatous, isoattenuation in solid and sclerotic and low attenuation in cystic areas. The most common CT signs are a marginal pseudocapsule (50\%), overlying vessel (26.3\%), halo sign (17.1\%) and air-gap sign (2.6\%). PSPs also frequently exhibit the tail sign on CT. When one or more of these signs are present, clinicians should be more alert to the possibility of PSP. The majority of reports suggest that PSPs are hypo or intermediate grade lesions on positron emission tomography (PET). A robotic right lower lobectomy was successfully performed in this case and allowed for the early discharge of the patient and a return to usual activities within 3 weeks. Minimally invasive thoracic surgery via a robotic approach is beneficial to both young, fit patients and may be also advantageous in those with poor lung function or multiple co-morbidities. The challenge with such cases is often with obtaining the correct preoperative radiological and histological diagnosis. Whilst no follow-up guideline for these rare tumours exists, in view of reported malignant potential we advocate close follow-up of patients.
\end{abstract}

Keywords: Robotic assisted surgery; minimally invasive surgery; pulmonary sclerosing pneumocytoma (PSP); case report

Received: 08 March 2021; Accepted: 30 May 2021; Published: 30 September 2021.

doi: $10.21037 /$ asj-21-11

View this article at: https://dx.doi.org/10.21037/asj-21-11

\section{Introduction}

Pulmonary sclerosing hemangioma was first described by Liebow and Hubbell in 1956 (1). The neoplasm was originally thought to be vascular in origin however, based on immunohistochemical studies, it is now thought to be epithelial in nature and was therefore renamed sclerosing pneumocytoma by the World Health Organization in 2015 (2).

Surgical resection is the treatment of choice of this rare lesion. This is carried out most commonly by wedge resection, segmentectomy or lobectomy. We present the

^ ORCID: 0000-0003-0672-1127. 


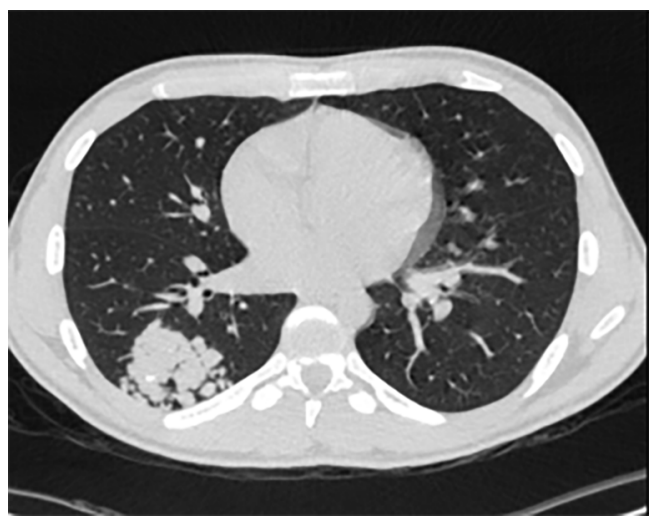

Figure 1 Axial slice CT thorax demonstrating right lower lobe lung mass. CT guided biopsy revealed a benign pulmonary sclerosing pneumocytoma.

following article in accordance with the CARE reporting checklist (available at https://asj.amegroups.com/article/ view/10.21037/asj-21-11/rc).

\section{Case presentation}

A 20-year-old Caucasian male suffered right chest wall trauma during a football match. He attended the emergency department where CT thorax demonstrated a posterolateral 9th right rib fracture, along with an incidental multilobulated $48 \mathrm{~mm}$ mass in the posterior aspect of the right lower lobe containing specks of calcium (Figure 1).

Full staging CT thorax abdomen and pelvis 3 days later revealed no other significant findings and there was no lymphadenopathy evident. The patient was referred to the lung multi-disciplinary meeting. PET-CT followed and demonstrated the lung mass to be PET avid with an SUV of 3.3 (mediastinal blood pool 1.9) and with no other uptake of significance. CT guided lung biopsy was then performed, with 2 cores taken using an $18 \mathrm{~g}$ co-axial needle. The pathologist reported this as a benign pulmonary sclerosing pneumocytoma (PSP) and, following further lung MDT discussion, the patient was seen in the thoracic clinic.

On assessment in the thoracic clinic, he was now asymptomatic. Past medical history consisted of a tonsillectomy at age 4 , asthma and the recent right $9^{\text {th }}$ rib fracture. He took no regular medication and was a never smoker. Examination was unremarkable. Pulmonary function testing demonstrated his forced expiratory volume in one second (FEV1) to be $100 \%$ predicted and diffusing capacity of the lung for carbon monoxide (DLCO) to be $102 \%$ predicted. The patient was counseled on the diagnosis and the management options discussed and risks and benefits of surgery. After electing to some time to consider the information, the patient decided to proceed with surgical resection. CT thorax was repeated 3 months after the last CT, prior to listing for resection. The right lower lobe mass had increased in size to a maximum diameter of $52 \mathrm{~mm}$ without any other significant findings.

The patient underwent a robotic right lower lobectomy uneventfully using the Intuitive Da Vinci Xi surgical system (Intuitive Surgical Inc, Sunnyvale, Calif.). A total of 4 ports were utilised, with 3 of $8 \mathrm{~mm}$ and one of $12 \mathrm{~mm}$. The surgical strategy started with dissecting the pulmonary artery branches in the oblique fissure. The anterior and posterior fissures were then completed with the robotic stapler. The apical segmental and basal trunk arteries were then also divided with the robotic stapler followed by the lower lobe bronchus and inferior pulmonary vein respectively. Lymph node sampling was performed, a 24F apical drain placed and the ports sutured closed in layers.

Total drainage on the first post-operative day was $55 \mathrm{~mL}$ with no air leak and the chest drain was removed. The patient was discharged from hospital on the second post-operative day.

Post-operative histology confirmed the mass to be a $54 \mathrm{~mm}$ sclerosing pneumocytoma with complete resection. The mass comprised a neoplastic proliferation of two populations of monomorphic epithelial cells. The first comprising cuboidal cells with acinar and papillary architecture. The second comprising less distinct cells with eosinophilic cytoplasm and vesicular nuclei occupying the stroma over and within the first population. Immunohistochemistry revealed expression of epithelial membrane antigen (EMA) by both populations but of transcription termination factor 1 (TTF1) by only the first.

The patient went on to make a good post-operative recovery, albeit with a course of antibiotics for a superficial wound infection. He remains under routine follow-up in our thoracic clinic.

All procedures performed in this study were in accordance with the ethical standards of the institutional and/or national research committee (s) and with the Helsinki Declaration (as revised in 2013). Written informed consent was obtained from the patient for publication of this case report and accompanying images. A copy of the written consent is available for review by the editorial office of this journal. 


\section{Discussion}

Our case describes a more unusual presentation of a PSP, in that it was found in a young Caucasian male. A large series from Shanghai suggests that PSP are most commonly found in middle age (40-70 years) females that are nonsmokers (3). The mass was more typical in terms of its location and less commonly PSP's can arise from pulmonary hilar region (4) or even have an endobronchial location (5). Whilst most typically present as a solitary peripheral nodule as we report here, rare cases with multiple PSP lesions have been reported (6).

The differential diagnosis of the CT and PET findings is wide and would include malignant causes such as non-small cell lung cancer, small cell lung cancer, carcinoid tumours and metastases. Non-malignant differentials would include congenital causes such as arteriovenous malformations or sequestrations as well as acquired infective, inflammatory, granulomatous lesions and non-malignant tumours such as hamartomas.

Regarding the radiological appearance of PSPs, they have been reported to be a solitary lesion in $92.1 \%$ cases, with a predominantly smooth boundary and oval shape (7). This may aid differentiation from the classically irregular and spiculated malignant pulmonary lesion. The varying proportions of the haemangiomatous, papillary, sclerotic and solid histological patterns result in varying CT density of 96 to 157 Hounsfield Units, with higher attenuation in angiomatous, isoattenuation in solid and sclerotic and low attenuation in cystic areas $(8,9)$. This would be distinct from the focal collections of fat aernating with areas of calcification within a hamartoma (10). It has been reported that PSPs are predominantly juxta pleural on axial CT (9), however this is not consistent across the literature with other groups reporting close to an equal mix of intraparenchymal and juxta-pleural location (7). The most common CT signs in a large retrospective review was marginal pseudocapsule (50\%), with lower frequencies of some other common radiological signs of overlying vessel (26.3\%), halo sign (17.1\%) and air-gap sign (2.6\%). PSPs also frequently exhibit the tail sign on CT (11). When one or more of these signs are present, clinicians should be more alert to the possibility of PSP.

A varying level of avidity on PET scan may be seen with these lesions, with tumour size and clinical features being associated with the level of uptake (12). It is worth noting that the majority of reports suggest that PSPs are hypo or intermediate grade lesions on $\operatorname{PET}(7,13,14)$. This may exclude any highly avid malignant, inflammatory or infective lesions from the radiological differential. The uptake of PSP is also higher than that of a pulmonary hamartoma (15).

The challenging nature of radiological diagnosis therefore makes a tissue diagnosis of PSP highly valuable. PSP can be diagnosed via preoperative endobronchial ultrasound-guided transbronchial needle aspiration (EBUSTBNA) but may prove a diagnostic challenge via fine needle aspiration (FNA) (16). In terms of immunostaining, the alveolar type 2-like cells stain positive for napsin A and round cells for vimentin. As the tumour is derived from primitive epithelial cells it expresses TTF-1 and a key histological feature is the presence of both cuboidal surface cells and stromal round cells (2). This results in the challenge in diagnosing them on frozen section, small biopsies and cytology where they can be easily mistaken for adenocarcinoma or carcinoid tumours $(2,17)$. A rate of accurate diagnosis of just $44 \%$ on frozen section emphasizes this point (17) and a deferral rate of $31 \%$ raises the risk of overtreatment (18). We believe this limits the benefit of utilizing a strategy of intra-operative frozen section as a means of diagnosis and going on to perform sublobar parenchymal sparing resection at the same sitting.

PSP is generally thought to be benign but in some cases malignant like behavior has been reported, with the presence of lymph node metastasis (19). It is therefore possible that a more aggressive phenotype exists. A published case describes the event where a patient had initially declined resection and this resulted in a delayed re-presentation with spinal metastases (20). Surgical resection is the treatment of choice and close follow-up thereafter is advised.

In or case, the robotic approach provided a means to an accelerated recovery for the patient and allow for a rapid return to daily routine. Whilst our patient was not currently working, he was able to return to playing football within 3 weeks of surgery. Utilising a less traumatic robotic approach, with minimal blood loss and post-operative pain, was beneficial for our young patient. We feel robotic surgery in this case did deliver these benefits and as such could be further utilised. The relative benefit compared to utilising a VATS approach must be determined, and we look forward to further evidence, including cost analyses, in this area, Robotic surgery is also likely to be advantageous for patients with poor lung function or comorbidities, where more invasive methods are undesirable. It would also be well-suited to cases of PSP where segmentectomy or wedge resection may be appropriate. In 
this case, based on the radiological assessment of tumour position and its size of over $5 \mathrm{~cm}$, sublobar resection was not feasible. Subsequent histological analysis confirmed a maximum tumour diameter of $53 \mathrm{~mm}$.

Whilst no follow-up guideline for these rare tumours exists, in view of reported malignant potential we advocate close follow-up of these patients. In this case, the patient was seen 2 weeks following surgery then every 3 months over the first year with a surveillance CT being performed at 12 months.

\section{Conclusions}

Here we report the first excision of a PSP via robotic technique. Robotic excision of PSP appears feasible and safe and as such can be further utilised. It is challenging to obtain a radiological diagnosis and confirmation via frozen section is not always possible. Mid and long-term outcomes are awaited.

\section{Acknowledgments}

Funding: None.

\section{Footnote}

Reporting Checklist: The authors have completed the CARE reporting checklist. Available at https://asj.amegroups.com/ article/view/10.21037/asj-21-11/rc

Conflicts of Interest: All authors have completed the ICMJE uniform disclosure form (available at https://asj.amegroups. com/article/view/10.21037/asj-21-11/coif). The authors have no conflicts of interest to declare.

Ethical Statement: The authors are accountable for all aspects of the work in ensuring that questions related to the accuracy or integrity of any part of the work are appropriately investigated and resolved. All procedures performed in this study were in accordance with the ethical standards of the institutional and/or national research committee (s) and with the Helsinki Declaration (as revised in 2013). Written informed consent was obtained from the patient for publication of this case report and accompanying images. A copy of the written consent is available for review by the editorial office of this journal.

Open Access Statement: This is an Open Access article distributed in accordance with the Creative Commons Attribution-NonCommercial-NoDerivs 4.0 International License (CC BY-NC-ND 4.0), which permits the noncommercial replication and distribution of the article with the strict proviso that no changes or edits are made and the original work is properly cited (including links to both the formal publication through the relevant DOI and the license). See: https://creativecommons.org/licenses/by-nc-nd/4.0/.

\section{References}

1. Liebow AA, Hubbell DS. Sclerosing hemangioma (histiocytoma, xanthoma) of the lung. Cancer 1956;9:53-75.

2. Travis WD, Brambilla E, Nicholson AG, et al. The 2015 World Health Organization Classification of Lung Tumors: Impact of Genetic, Clinical and Radiologic Advances since the 2004 Classification. J Thorac Oncol 2015;10:1243-60.

3. Zhu J. Analysis of the clinical differentiation of pulmonary sclerosing pneumocytoma and lung cancer. J Thorac Dis 2017;9:2974-81.

4. Hung JH, Hsueh C, Liao CY, et al. Pulmonary Hilar Tumor: An Unusual Presentation of Sclerosing Hemangioma. Case Rep Med 2016;2016:8919012.

5. Khanna A, Alshabani K, Mukhopadhyay S, et al. Sclerosing pneumocytoma. Medicine (Baltimore) 2019;98:e15038.

6. Fan X, Lin L, Wang J, et al. Genome profile in a extremely rare case of pulmonary sclerosing pneumocytoma presenting with diffusely-scattered nodules in the right lung. Cancer Biol Ther 2018;19:13-9.

7. Shin SY, Kim MY, Oh SY, et al. Pulmonary sclerosing pneumocytoma of the lung: CT characteristics in a large series of a tertiary referral center. Medicine (Baltimore) 2015;94:e498.

8. Chung MJ, Lee KS, Han J, et al. Pulmonary Sclerosing Hemangioma Presenting as Solitary Pulmonary Nodule: Dynamic CT Findings and Histopathologic Comparisons. AJR Am J Roentgenol 2006;187:430-7.

9. Wang QB, Chen YQ, Shen JJ, et al. Sixteen cases of pulmonary sclerosing haemangioma: CT findings are not definitive for preoperative diagnosis. Clin Radiol 2011;66:708-14.

10. Siegelman SS, Khouri NF, Scott WW, et al. Pulmonary hamartoma: CT findings. Radiology 1986;160:313-7.

11. Cheung YC, Ng SH, Chang JWC, et al. Histopathological and CT features of pulmonary sclerosing haemangiomas. Clin Radiol 2003;58:630-5.

12. Xu J, Dong Y, Yin G, et al. 18F-FDG PET/CT imaging: 
A supplementary understanding of pulmonary sclerosing pneumocytoma. Thorac Cancer 2019;10:1552-60.

13. Hara M, Iida A, Tohyama J, et al. FDG-PET findings in sclerosing hemangioma of the lung: A case report. Radiat Med 2001;19:215-8.

14. Timpone V, Danielson D, Woods A, et al. FDG-PET imaging findings of a pulmonary sclerosing hemangioma. Eur J Radiol Extra 2011;79:e65-7.

15. Jiang L, Huang Y, Tang Q, et al. 18 F-FDG PET/CT characteristics of pulmonary sclerosing hemangioma vs. pulmonary hamartoma. Oncol Lett 2018;16:660-5.

16. Zeng J, Zhou F, Wei XJ, et al. Sclerosing hemangioma: A diagnostic dilemma in fine needle aspiration cytology.

doi: $10.21037 /$ asj-21-11

Cite this article as: Smith MO, Mason SL, Duvva D, Woolley SM. Robotic excision of pulmonary sclerosing pneumocytoma: a case report. AME Surg J 2021;1:19.
Cytojournal 2016;13:9.

17. Hissong E, Rao R. Pneumocytoma (sclerosing hemangioma), a potential pitfall. Diagn Cytopathol 2017;45:744-9.

18. Low SY, Teo F, Eng P, et al. Pulmonary sclerosing hemangioma: Pitfalls in management. Asian Cardiovasc Thorac Ann 2011;19:139-42.

19. Xu HM, Zhang G. A rare case of pulmonary sclerosing hemagioma with lymph node metastasis and review of the literature. Int J Clin Exp Pathol 2015;8:8619-23.

20. Kim MK, Jang SJ, Kim YH, et al. Bone metastasis in pulmonary sclerosing hemangioma. Korean J Intern Med 2015;30:928-30. 